\title{
Hypoalbuminemia as a relapse risk factor after high dose chemotherapy and autologous stem cell transplantation in patients with Hodgkin's lymphoma
}

\section{Paweł Koźlik', Monika Długosz-Danecka², Renata Pacholczak³, Agata Bryk ${ }^{4}$, Agata Krawczyk ${ }^{5}$, Wojciech Jurczak ${ }^{2}$}

'Department of Allergy and Immunology, Jagiellonian University Medical College, Cracow, Poland

${ }^{2}$ Department of Hematology, Jagiellonian University Medical College, Cracow, Poland

${ }^{3}$ Department of Anatomy, Jagiellonian University Medical College, Cracow, Poland

${ }^{4}$ Institute of Cardiology, Jagiellonian University Medical College, Cracow, Poland

${ }^{5}$ Department of Anatomy, Jagiellonian University Medical College, Cracow, Poland

Correspondence:

Monika Długosz-Danecka Department of Hematology,

Jagiellonian University

Medical College, Cracow

31-501 Cracow, Kopernika 17

e-mail:

monika.dlugosz-danecka@uj.edu.pl

Received:

19.11.2018

Accepted:

10.12.2018

DOI: 10.24292/01.OR.101218 Copyright $\odot$ Medical Education. All rights reserved.

\section{ABSTRACT}

Assessing the risk factors according to EORTC (European Organization of Research and Treatment of Cancer) or HD IPS (Hodgkin's lymphoma International Prognostic Score) is crucial for choosing the adequate I line therapy in Hodgkin's lymphoma patients. However, none of those scales predicts the efficacy of autologous stem cell transplant (ASCT) in relapsing/refractory (R/R) patients. Progression-free survival (PFS) after ASCT corelates with the response to salvage regimens. Thirty-nine Hodgkin's lymphoma patients transplanted at Jagiellonian University Department of Hematology with a follow-up exceeding 5 years were analyzed. Despite long time periods since the diagnosis, initial serum albumin levels seem to correlate with progression after autologous stem cell transplant and indicate high probability of relapse within 5 months following the procedure with favorable test characteristics.

Key words: hypoalbuminemia, Hodgkin's lymphoma, risk factors 


\section{INTRODUCTION}

Hodgkin's lymphoma is a disease with a favorable outcome. Only minority of the patients, relapsing or refractory $(R / R)$ after the first line chemoradiotherapy, remain a therapeutic challenge. The salvage regimens followed - in responding patients - by autologous stem cell transplant (ASCT) consolidation - are the next step in curative approach. The more intense the induction regimen, the fewer patients require salvage therapy, although it is usually less effective. Novel biological therapies such as brentuximab or nivolumab are the current standard of care in refractory cases and those relapsing after ASCT. They prolong overall survival (OS) and - in selected patients - serve as a bridging to potentially curative allogeneic stem cell transplantation [1]. In our study we focused on defining risk factors present at diagnosis, predicting relapse after ASCT, in attempt to select candidates for potential preemptive therapy with novel biological agents.

\section{MATERIALS AND METHODS}

\section{Study design}

A single-center retrospective study based on local patients' registry. Patients diagnosed with histologically-proven nodular sclerosis variant of Hodgkin's lymphoma (HD), clinical stages (CS) IIBX-IV, after autologous stem cell transplantation performed at the Jagiellonian University Department of Hematology between January 1999 and August 2012 were analyzed. Initial risk factor assessment included clinical staging performed in accordance to Cotswold's-modified Ann Arbor classification, data acquired from patient's history, physical examination, unilateral iliac bone marrow biopsy, chest and abdomen computed tomography (CT) scans and laboratory studies: complete blood count, erythrocyte sedimentation rate (ESR), hemoglobin $(\mathrm{Hb})$, albumin, and lactate dehydrogenase levels (LDH). Both prognostic indexes: EORTC (European Organization for Research and Treatment of Cancer) [2] and HD IPS (International Prognostic Index) [3] were calculated. These data as well as chemotherapy protocols and indications for ASCT were analyzed in search for risk factors of relapse after ASCT.

\section{Treatment protocol}

Outside clinical trials, in a real-life setting at the Jagiellonian University Department of Hematology, we used the strategy to initiate treatment with ABVD (doxorubicin, bleomycin, vinblastine, dacarbazine) or BEACOPP (etoposide, doxorubicin, cyclophosphamide, procarbazine, bleomycin, vincristine, prednisone) chemotherapy regimen depending on risk factors and patient's informed choice. It was followed by early positron emission to- mography (PET) response assessment after $2^{\text {nd }}$ chemotherapy cycle and obligatory therapy modification (maintaining, escalating or de-escalating treatment intensity). Patients with complete response (CR) as indicated by PET were further treated with ABVD, regardless of the choice of the initial regimen, while the therapy of patients with persisting active disease was changed to escalated BEACOPP or salvage regimens (ESHAP or IGEV) followed by BEAM (carmustine, etoposide, cytarabine, melphalan)-conditioned autologous stem cell transplants. Regimens' dosing was based on their original description [4, 5]. Restaging and evaluation of treatment was performed in concordance with original Cheson's response criteria [6].

\section{End-points}

We developed a prognostic model based on patients' characteristics at diagnosis, validated by the outcome after ASCT. Relapse-free survival (RFS) following ASCT was defined as time from the transplant procedure to relapse, death or the end of observation. Event date was based on medical history, clinical examination or medical imaging studies (CT or PET), whichever occurred earlier. Clinical signs of relapse were defined as new-onset B-type symptoms or findings in physical examination (e.g. new lymph node enlargement), if they were subsequently confirmed by imaging studies. OS was defined as time from diagnosis to patient's death from any cause or end of observation.

\section{Statistical analysis}

Relapse-free survival and OS were assessed using Kaplan-Meier's statistics and compared by Cox's F test to compensate for small sample size. Patient's demographics, treatment protocols preceding ASCT, indications for ASCT, initial clinical stage, IPS and EORTC scores for HD as well as particular parameters of those scales were exercised in search for factors of relapse. Prognostic model developed using logistic regression analysis was cross-validated on randomly divided sample to calculate its test characteristics.

\section{RESULTS}

A total of 39 patients treated at our center met the inclusion criteria (tab. 1). The majority had ASCT due to primary resistance to chemotherapy ( $n=17 ; 44 \%)$, followed by those with relapse $(n=12 ; 31 \%)$ and consolidation of initial treatment after complete response $(n=10 ; 25 \%)$. As much as $87 \%$ (34 of 39) of transplant recipients remained in remission, with median observation time of 60 months. Five patients (13\%) relapsed, with median time to relapse of 4.5 months. All of the relapsing patients had ASCT due to primary resistance. 
TABLE 1.

Sample characteristics.

\begin{tabular}{|c|c|c|c|c|}
\hline Characteristic & All groups & Remission & Relapse & $\begin{array}{c}\text { Difference } \\
\text { p-value }\end{array}$ \\
\hline No. of patients (\% total) & $39(100 \%)$ & $34(87 \%)$ & $5(13 \%)$ & - \\
\hline Sex (\% male) & $49 \%$ & $50 \%$ & $40 \%$ & $0.68^{*}$ \\
\hline Age (years, median) & 25.0 & 24.8 & 25.0 & $0.63^{* *}$ \\
\hline Clinical stage (mode) & CS IV & CS II BX, CS IV & CS IV & $0.20^{*}$ \\
\hline EORTC (pts, mode) & 3.0 & 3 & $2 ; 3$ & $0.12^{*}$ \\
\hline HD IPS (pts, mode) & 2.0 & 2 & $2 ; 3$ & $0.67^{*}$ \\
\hline
\end{tabular}

* Pearson's chi-square test.

*** Mann-Whitney U test.

Of the analyzed risk factors (tab. 2, 3), only low serum albumin concentration at diagnosis was found to distinguish those with unfavorable outcome. Serum albumin level was thus included in logistic regression analysis to estimate cutoff value for increased risk. In our sample, the probability of relapse was given by the following equation:

$$
P_{\text {relapse }}=\frac{e^{115,73-3,27[\text { serumalbumin }]}}{1+e^{115,73-3,27[\text { serum albumin }]}}
$$

The cutoff value of albumin level increasing probability of relapse beyond $50 \%$ estimated using the given formula is $35.4 \mathrm{~g} / \mathrm{l}$, the lower reference limit of serum albumin concentration (fig. 1). The cutoff value significantly distinguished between PFS among ASCT recipients (fig. 2, 3). Model's positive predictive value (PPV) is estimated at $0.39-1.0(95 \% \mathrm{Cl}$ [confidence interval]), and negative predictive value (NPV) at $0.83-0.99(95 \% \mathrm{Cl})$, with sensitivity at $0.29-0.98$ and specificity at 0.87-1.0.

TABLE 2.

Predictors of relapse following ASCT (multiple logistic regression analysis).

\begin{tabular}{|c|c|c|c|c|}
\hline Analyzed factor & Value & Adjusted odds ratio (AOR) & $95 \% \mathrm{Cl}$ & p-value \\
\hline \multirow[t]{2}{*}{$1^{\text {st }}$ line chemotherapy } & BEACOPP & 0.45 & $0.5-5.3$ & 0.52 \\
\hline & ABVD & $1^{*}$ & - & - \\
\hline \multirow[t]{2}{*}{$2^{\text {nd }}$ line chemotherapy } & BEACOPP & 0.47 & $0.4-2.8$ & 0.41 \\
\hline & ABVD & $1^{*}$ & - & - \\
\hline \multirow[t]{3}{*}{ Clinical stage } & IV & 0.47 & $0.01-6.4$ & 0.61 \\
\hline & III & 0.77 & $0.04-15.1$ & 0.93 \\
\hline & II BX & $1^{*}$ & - & - \\
\hline Age & (unit change) & 1.03 & $0.9-1.1$ & 0.54 \\
\hline \multirow[t]{2}{*}{ Sex } & Female & & & \\
\hline & Male & $1^{*}$ & - & - \\
\hline EORTC & (unit change) & 0.75 & $0.2-3.1$ & 0.69 \\
\hline HD IPS & (unit change) & 0.4 & $0.1-2.7$ & 0.33 \\
\hline
\end{tabular}

* Reference value.

TABLE 3.

Individual parameters at diagnosis and odds of relapse following ASCT (multiple logistic regression analysis).

\begin{tabular}{|c|c|c|c|c|}
\hline Analyzed factor (median) & All groups & Remission & Relapse & p-value \\
\hline Albumin level (g/l) & 41.5 & 43 & 35.0 & $<0.01$ \\
\hline $\operatorname{ESR}(\mathrm{mm} / \mathrm{h})$ & 50 & 49 & 63.0 & 0.42 \\
\hline $\mathrm{LDH}(\mathrm{U} / \mathrm{I})$ & 440 & 442 & 407 & 0.89 \\
\hline Platelet count $\left({ }^{*} 10^{9} / \mathrm{l}\right)$ & 420 & 431 & 317 & 0.35 \\
\hline Lymphocyte count $\left({ }^{*} 10^{9} / \mathrm{I}\right)$ & 0.16 & 0.18 & 0.16 & 0.69 \\
\hline White blood cell count $\left({ }^{*} 10^{9} / \mathrm{I}\right)$ & 13 & 12.5 & 13.0 & 0.68 \\
\hline $\mathrm{Hb}(\mathrm{g} / \mathrm{dl})$ & 11.9 & 12.0 & 11.0 & 0.12 \\
\hline Mediastinal mass maximal diameter (cm) & 6.5 & 6.5 & 7.8 & 0.92 \\
\hline
\end{tabular}


FIGURE 1.

Borderline serum albumin level as estimated using logistic regression analysis. Rhombus size expresses number of patients.

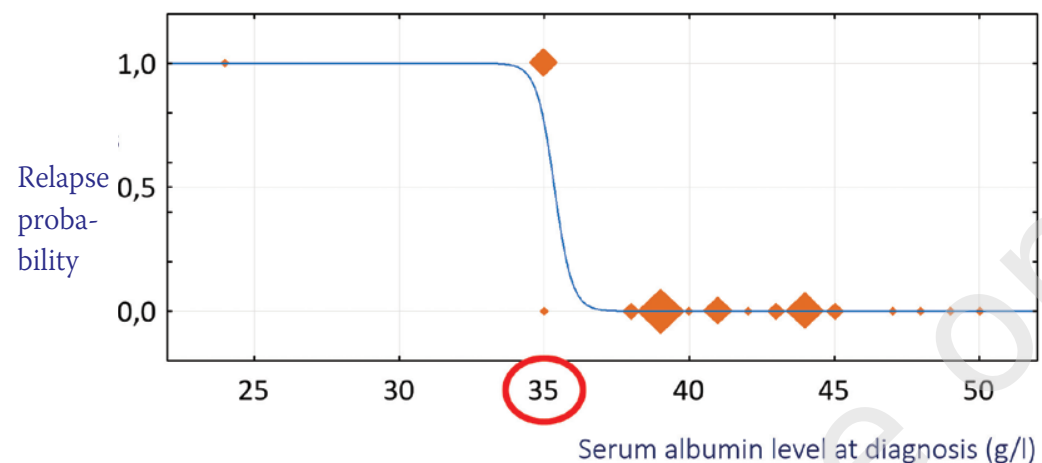

FIGURE 2.

Progression-free survival following ASCT, in HD patients with normal vs low albumin at diagnosis.

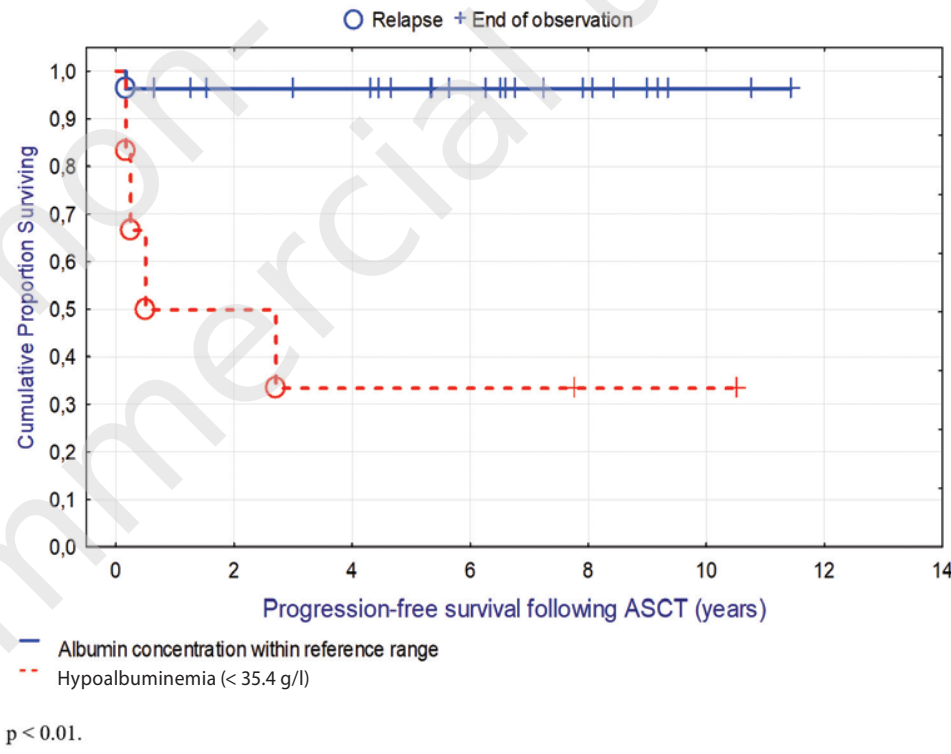

FIGURE 3.

Overall survival following ASCT, in HD patients with normal vs low albumin at diagnosis.

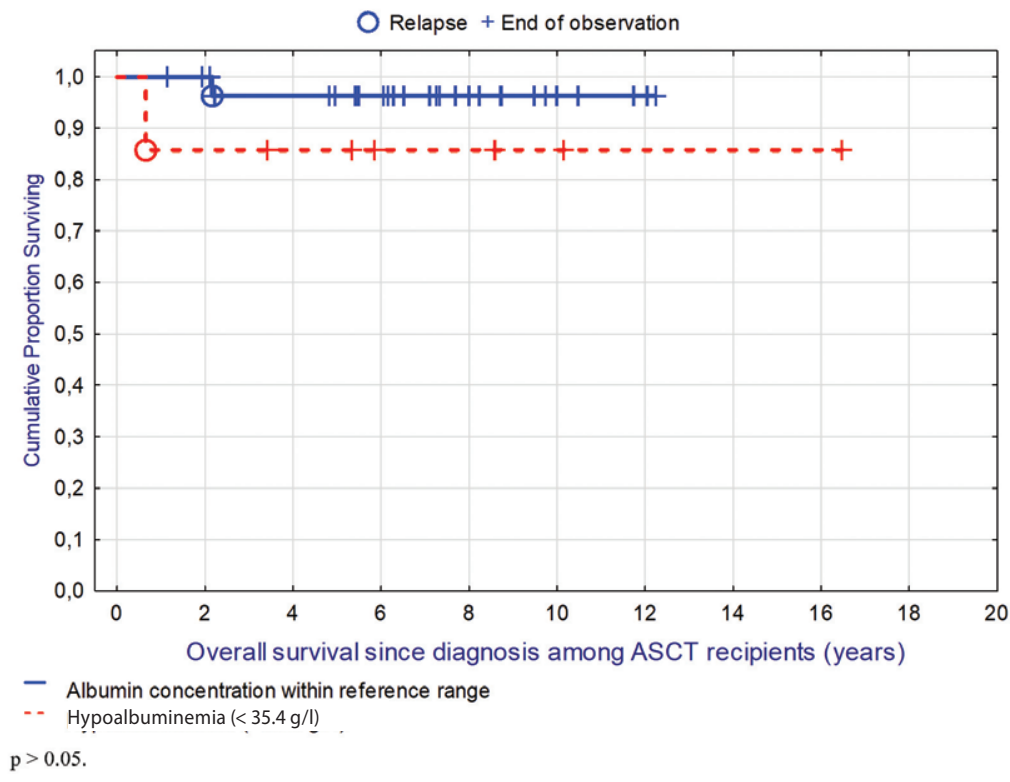




\section{DISCUSSION}

The more intensive the first line regimen in $\mathrm{HD}$, the less effective the salvage in $\mathrm{R} / \mathrm{R}$ patients. Although only few patients relapse or are refractory to escalated BEACOPP, they are rarely chemosensitive to salvage chemotherapy, further consolidated by ASCT. Similarly, the more the chemotherapy lines, and the worse the response to salvage, the worse prognosis [7]. Achieving a PET confirmed CR is laterally doubles post-transplant PFS [8].

Our data suggest that a low albumin level at diagnosis identifies patients who frequently relapse after ASCT. Other risk factors present at diagnosis do not have distinct prognostic value at the time of transplant [9]. Hypoalbuminemia in Hodgkin's lymphoma may be linked to elevated IL-10 levels [10] which was already suggested as an independent prognostic factor with an unfavorable outcome [11]. The biological explanation of these findings remains uncertain. If our conclusions are confirmed in the larger cohort of patients, low albumin levels at diagnosis may be an important prognostic factor, selecting the candidates for targeted therapy.

How does our observation fit to HD therapy landscape? There are variety of therapeutic options for R/R HD patients. Previous PLRG (Polish Lymphoma Research Group) analysis from 2002 [12], performed before the introduction of targeted therapy, recommended ASCT even in high risk patients, with a minor response to salvage regimens. Although none of the chemo-only regimens was ever compared to the others in a randomized III phase study, classical cisplatin-based chemotherapy combinations (ICE, ESHAP, DHAP) are being gradually replaced by poten- tially less toxic gemcitabine combinations. Among those particularly commonly used, one has to mention IGEV (ifosfamide, gemcitabine, vinorelbine) [13] and BeGeV (bendamustine, gemcitabine, vinorelbine) [14]. PLRG is currently investigating efficacy of BGD (bendamustine, gemcitabine, dexamethasone) in patients refractory to ABVD induction therapy. The results of chemo-only regimens are clearly inferior to brentuximab vedotin (BV) used in monotherapy [15] or in combinations with i.e. bendamustine $[16,17]$. BV is the anti CD30 monoclonal antibody, combined with the cellular toxin (auristatin), registered in R/R HD. Originally recommended in patients relapsing after ASCT, given its high efficacy to "bridge" patients to allo-SCT (allogeneic stem cell transplantation). At present it would be also considered in patients not responding well to the initial salvage therapy, before ASCT [18]. In Hodgkin lymphoma patients with low albumin at diagnosis, not achieving a PET confirmed CR after the salvage therapy, BV could therefore further decrease the tumor burden and sensitize them to ASCT conditioning regimen.

\section{CONCLUSIONS}

Despite long time periods since the diagnosis, initial serum albumin levels seem to correlate with progression after autologous stem cell transplant and indicate high probability of relapse within 5 months following the procedure with favorable test characteristics. Alternative targeted therapies, like BV should be considered in R/R HD patients with low albumin at diagnosis, not achieving $C R$ after salvage regimens, regarding high relapse rate observed.

\section{References}

1. Stathis A, Younes A. The new therapeutical scenario of Hodgkin lymphoma. Ann Oncol 2015; 26(10): 2026-2033. DOI: 10.1093/annonc/mdv256.

2. Klimm B, Goergen H, Fuchs $M$ et al. Impact of risk factors on outcomes in early-stage Hodgkin's lymphoma: an analysis of international staging definitions. Ann Oncol 2013; 24(12): 3070-3076. DOI: 10.1093/annonc/mdt413.

3. Hasenclever D, Diehl V. A prognostic score for advanced Hodgkin's disease. International Prognostic Factors Project on Advanced Hodgkin's Disease. N Engl J Med 1998; 339(21): 1506-1514. DOI: 10.1056/NEJM199811193392104.

4. DiehI V, Franklin J, Pfreundschuh M et al. Standard and increased-dose BEACOPP chemotherapy compared with COPP-ABVD for advanced Hodgkin's disease. N Engl J Med 2003; 348(24): 2386-2395. DOI: 10.1056/NEJMoa022473.

5. Bonadonna G, Zucali R, Monfardini S et al. Combination chemotherapy of Hodgkin's disease with adriamycin, bleomycin, vinblastine, and imidazole carboxamide versus MOPP. Cancer 1975; 36(1): 252-259.

6. Cheson BD, Pfistner B, Juweid ME et al. Revised response criteria for malignant lymphoma. J Clin Oncol 2007; 25(5): 579-586. DOI: 10.1200/ JCO.2006.09.2403.

7. Czyz J, Dziadziuszko R, Knopinska-Postuszuy W et al. Outcome and prognostic factors in advanced Hodgkin's disease treated with high-dose chemotherapy and autologous stem cell transplantation: a study of 341 patients. Ann Oncol 2004; 15(8): 1222-1230. DOI: 10.1093/annonc/mdh304.

8. Moskowitz CH, Matasar MJ, Zelenetz AD et al. Normalization of pre-ASCT, FDG-PET imaging with second-line, non-cross-resistant, chemotherapy programs improves event-free survival in patients with Hodgkin lymphoma. Blood 2012; 119(7): 1665-1670. DOI: 10.1182/blood-2011-10-388058.

9. Chopra R, McMillan AK, Linch DC et al. The place of high-dose BEAM therapy and autologous bone marrow transplantation in poor-risk Hodgkin's disease. A single-center eight-year study of 155 patients. Blood 1993; 81(5): 1137-1145.

10. Gaiolla RD, Domingues MA, Niero-Melo L et al. Serum levels of interleukins 6, 10, and 13 before and after treatment of classic Hodgkin lymphoma. Arch Pathol Lab Med 2011; 135(4): 483-489. DOI: 10.1043/2010-0060-OA.1. 
11. Bohlen $\mathrm{H}$, Kessler M, Sextro $M$ et al. Poor clinical outcome of patients with Hodgkin's disease and elevated interleukin-10 serum levels. Clinical significance of interleukin-10 serum levels for Hodgkin's disease. Ann Hematol 2000; 79(3): 110-113.

12. Czyz J, Hellmann A, Dziadziuszko R et al. High-dose chemotherapy with autologous stem cell transplantation is an effective treatment of primary refractory Hodgkin's disease. Retrospective study of the Polish Lymphoma Research Group. Bone Marrow Transplant 2002; 30(1): 29-34. DOI: 10.1038/sj.bmt.1703590.

13. Santoro A, Magagnoli M, Spina M et al. Ifosfamide, gemcitabine, and vinorelbine: a new induction regimen for refractory and relapsed Hodgkin's lymphoma. Haematologica 2007; 92(1): 35-41.

14. Santoro A, Mazza R, Pulsoni A et al. Bendamustine in Combination With Gemcitabine and Vinorelbine Is an Effective Regimen As Induction Chemotherapy Before Autologous Stem-Cell Transplantation for Relapsed or Refractory Hodgkin Lymphoma: Final Results of a Multicenter Phase II Study. J Clin Oncol 2016; 34(27): 3293-3299. DOI: 10.1200/JCO.2016.66.4466.

15. Chen R, Gopal AK, Smith SE et al. Five-year survival and durability results of brentuximab vedotin in patients with relapsed or refractory Hodgkin lymphoma. Blood 2016; 128(12): 1562-1566. DOI: 10.1182/blood-2016-02-699850.

16. O'Connor OA, Lue JK, Sawas A et al. Brentuximab vedotin plus bendamustine in relapsed or refractory Hodgkin's lymphoma: an international, multicentre, single-arm, phase 1-2 trial. Lancet Oncol 2018; 19(2): 257-266. DOI: 10.1016/S1470-2045(17)30912-9.

17. LaCasce AS, Bociek RG, Sawas A et al. Brentuximab vedotin plus bendamustine: a highly active first salvage regimen for relapsed or refractory Hodgkin lymphoma. Blood 2018; 132(1): 40-48. DOI: 10.1182/blood-2017-11-815183.

18. Broccoli A, Zinzani PL. The role of transplantation in Hodgkin lymphoma. Br J Haematol 2018. DOI: 10.1111/bjh.15639.

Authors' contributions:

Paweł Koźlik: performed the study and analyzed the data, had a substantial contribution to the conception and design of the work, drafted the manuscript, critically revised the manuscript for important intellectual content, had a substantial contribution to the acquisition, analysis and interpretation of data for the work

Monika Długosz-Danecka: performed the study and analyzed the data, had a substantial contribution to the conception and design of the work, drafted the manuscript, critically revised the manuscript for important intellectual content, had a substantial contribution to the acquisition, analysis and interpretation of data for the work Renata Pacholczak: performed the study and analyzed the data, had a substantial contribution to the acquisition, analysis and interpretation of data for the work; Agata Bryk: performed the study and analyzed the data, had a substantial contribution to the acquisition, analysis and interpretation of data for the work; Agata Krawczyk: performed the study and analyzed the data, had a substantial contribution to the acquisition, analysis and interpretation of data for the work;

Wojciech Jurczak: performed the study and analyzed the data, had a substantial contribution to the conception and design of the work, drafted the manuscript, critically revised the manuscript for important intellectual content, had a substantial contribution to the acquisition, analysis and interpretation of data for the work. Ethics:

The authors had full access to the data and take full responsibility for its integrity. All authors have read and agreed with the content of the manuscript as written. All authors declare no conflict of interest.

This work has been carried out in accordance with The Code of Ethics of the World Medical Association (Declaration of Helsinki) for experiments involving humans. 